\title{
Trans-abdominal ultrasonic findings correlated with CD4+ counts in adult HIV-infected patients in Benin, Nigeria
}

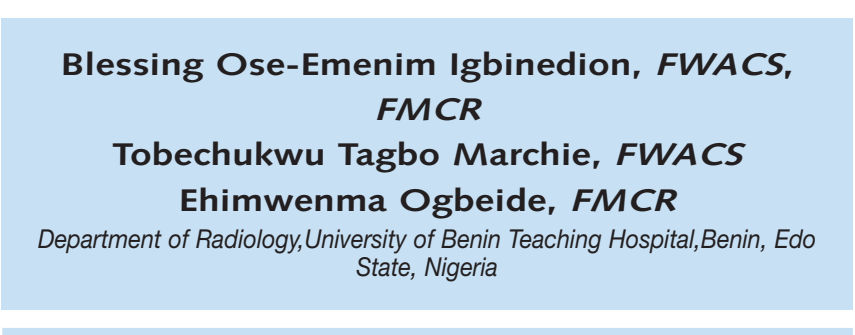

\section{Abstract}

Objective. The objective of this study was to document the abdominal ultrasound findings in HIV-infected patients and compare these with their $\mathrm{CD} 4+$ counts.

Patients and methods. Three hundred confirmed HIV-positive patients underwent abdominal ultrasonography at the University of Benin Teaching Hospital from November 2007 to January 2008. Each patient's sonographic findings were correlated with their CD4+ counts using the World Health Organization's HIV classification index.

Results. Splenomegaly, hepatomegaly, renomegaly, hyperechoic splenic parenchyma, increased renal echogenicity and lymphadenopathy are among the common sonographic findings. However, few of the ultrasound findings correlated statistically with the CD4+ counts.

Conclusion. The use of ultrasound as a baseline imaging modality in HIV-infected patients should be promoted. Its use is invaluable in the assessment of the disease state and in the monitoring of therapy and management of these immune-compromised individuals, who may have several abdominal presentations.

\section{Introduction}

HIV infection is a major public health concern worldwide, especially in sub-Saharan Africa, which currently has the highest morbidity and mortality. ${ }^{1}$ The first reported case of AIDS in Nigeria was in 1986, and Nigeria has a prevalence rate in the range of $3.6-8.0 \%$, depending on the locality. ${ }^{2}$

This cytopathic retrovirus destroys the immune system, leading to opportunistic infections and tumours. As a result, various organs of the body may be affected, leading to a variety of clinical presentations. Radiology plays a crucial role in the management of these patients. Radiologic tools (e.g. ultrasonography) serve both diagnostic and interventional roles, and assist in directing appropriate therapy. Computed tomography (CT) and magnetic resonance imaging (MRI) better characterise HIV-related abdominal diseases but are expensive and unavailable in the typically impoverished, HIV-infected sub-Saharan regions.

The degree of immunodeficiency is related to the level of the CD4+ count and, as such, CD4+ count is a good index for monitoring the disease's progression. ${ }^{3}$ It is expected that, as immune status decreases, susceptibility to infection and consequently abnormal sonographic findings should increase.

To the best of our knowledge, there is unfortunately a paucity of literature from West Africa (including Nigeria) on abdominal sonographic findings in HIV/AIDS patients; also, there is none which correlates such findings with patients' $\mathrm{CD} 4+$ counts.

\section{Method}

Abdominal ultrasounds scans were prospectively done on 300 consecutive adult patients (aged $\geq 18$ years) who were confirmed to have HIV/AIDS. The patients were recruited from the United States President's Emergency Plan for AIDS Relief (PEPFAR), and consultants' outpatients and general outpatients clinics of the University of Benin Teaching Hospital (UBTH). All the patients were aware of the existence of antiretroviral drugs and used these entry points to benefit from free HIV/AIDS treatment, which is available at this hospital.

Approval by the UBTH ethical committee for this study was granted. Informed written consent was given by the patients (all were conscious and fit to do so) before using their demographic and other data from their case notes, as well as for their participation in this ultrasonic study.

The ultrasound scanning machines used in this study were a Medison Sonoace-1500 (Korea, 1995) with $3.5 \mathrm{MHz}$ linear and 6.5 $\mathrm{MHz}$ curvilinear probes, and a Suis CTS-7700 (Shantou, China, 2007) with a $2.5-5.0 \mathrm{MHz}$ curvilinear probe which has variable focal zone and frequency capability. The high-frequency $(6.5 \mathrm{MHz})$ probe of the Sonoace machine was used to evaluate the gallbladder, appendix, anterior abdominal wall and other superficial structures; the Suis machine was used for the other abdominal organs. Proper setting of the overall (system) gain and the time or depth gain compensation (TGC/DGC) was adjusted to optimally visualise each organ.

To examine the various abdominal organs, patients lay on the examination couch in different postures which included supine, prone, lateral and oblique positions. The abdominal organs were scanned in longitudinal, transverse and oblique planes.

The following criteria were utilised to assess the abdominal organs: lymphadenopathy - visualised lymph nodes with the short axis measured; hepatomegaly - longitudinal dimension at mid-clavicular line $>15 \mathrm{~cm}$; splenomegaly - longitudinal dimension $>12 \mathrm{~cm}$; thickened gallbladder wall - dimension $>3 \mathrm{~mm}$ at the anterior wall; pancreatic enlargement - dimensions $>2.5 \mathrm{~cm}, 1.5 \mathrm{~cm}$ or $2.0 \mathrm{~cm}$ for the head, body or tail respectively; renomegaly - longitudinal renal dimension $>12$ $\mathrm{cm}$; thickened bowel wall - wall thickness $>4 \mathrm{~mm}$; biliary dilatation - intrahepatic biliary ducts luminal diameter $>2 \mathrm{~mm}$ (or if $>40 \%$ of the diameter of the adjacent portal vein) or extrahepatic biliary duct luminal 


\section{ORIGINAL ARTICLE}

\begin{tabular}{|lllll|}
\hline \multicolumn{5}{|c|}{ Table I. Age and sex distribution of the patients } \\
\hline Age & Male & Female & Total & Percent \\
\hline $18-27$ & 5 & 42 & 47 & 15.7 \\
$28-37$ & 28 & 95 & 123 & 41.0 \\
$38-47$ & 41 & 57 & 98 & 32.7 \\
$48-57$ & 9 & 13 & 22 & 7.3 \\
$58-67$ & 3 & 6 & 9 & 3.0 \\
$68-77$ & 0 & 1 & 1 & 0.3 \\
Total & 86 & 214 & 300 & 100 \\
& $(28.7 \%)$ & $(71.3 \%)$ & $(100 \%)$ & \\
\hline
\end{tabular}

\begin{tabular}{llll|}
$\begin{array}{l}\text { Table II. Normal/abnormal abdominal ultrasound scan v. } \\
\text { CD4+ classification }\end{array}$ \\
\hline $\begin{array}{l}\text { CD4+ class } \\
\text { N (\% within class) }\end{array}$ & Normal & Abnormal & Total \\
\hline Not significant & $5(14.3 \%)$ & $30(85.7 \%)$ & $35(100 \%)$ \\
Mild & $15(23.8 \%)$ & $48(76.2 \%)$ & $63(100 \%)$ \\
Advanced & $10(15.9 \%)$ & $53(84.1 \%)$ & $63(100 \%)$ \\
Severe & $14(10.1 \%)$ & $125(89.9 \%)$ & $139(100 \%)$ \\
$\quad$ Total & $44(14.7 \%)$ & $256(85.3 \%)$ & $300(100 \%)$ \\
Chi square $=6.630 ; p=0.085$. & & & \\
\hline
\end{tabular}

diameter at the porta hepatis $>5 \mathrm{~mm}$ for patients $<50$ years of age (or additional $1 \mathrm{~mm}$ per additional decade of life).

Data analysis was done using SPSS 13.0. Variables were expressed as percentages and comparison was by chi-square analysis. Two-tailed $p$ values $<0.05$ were considered significant.

\section{Results}

Two hundred and fourteen (71.3\%) of the patients were women and 86 (28.7\%) were men, with a female-to-male ratio of 2.5:1 (Table I). The age range of the patients was $21-70$ years (mean age $36.5+9$ years). Patients within the age group 28 - 37 years were the most common participants in this study.

\section{CD4 classification}

Grouping into CD4+ classes was done according to the World Health Organization's (WHO's) classification of CD4+ immunological profile in adult HIV-infected patients, with CD4+ counts $>500 /$ microlitre categorised into the None or Not significant class; 350 - 499 as Mild; 200 - 349 as Advanced; and a CD4+ count $<200$ in the Severe category. ${ }^{4}$ There were $35(11.7 \%)$ patients in the Not significant category, $63(21.0 \%)$ in the Mild, 63 (21.0\%) in the Advanced, and 139 (46.3\%) in the Severe CD4+ class.

The mean CD4+ count was $270 \pm 201 /$ microlitre. The minimum CD4+ value for females was $11 /$ microlitre and the maximum was 1000 microlitre, whereas the minimum value for males was $2 /$ microlitre and the maximum was $891 /$ microlitre. There were more women in each CD $4+$ class than males $(80.0 \%$ v. $20.0 \%, 68.3 \%$ v. $31.7 \%, 69.8 \%$ v. $30.2 \%$,

\begin{tabular}{|c|c|c|c|c|c|c|c|}
\hline \multicolumn{8}{|c|}{ Table III. Sonographic findings v. CD4+ classification } \\
\hline \multirow[t]{2}{*}{$\begin{array}{l}\text { Sonographic } \\
\text { findings }\end{array}$} & \multirow[t]{2}{*}{$\begin{array}{c}\text { Frequency } \\
(\%)\end{array}$} & \multicolumn{4}{|c|}{ CD4+ classification $\mathrm{N}(\%)$} & \multirow{2}{*}{$\begin{array}{c}\text { Chi-square } \\
\chi^{2}\end{array}$} & \multirow[t]{2}{*}{$p$ value } \\
\hline & & $\begin{array}{c}\text { Not } \\
\text { Significant }\end{array}$ & Mild & Advanced & Severe & & \\
\hline Splenomegaly & $133(44.3 \%)$ & $9(25.7 \%)$ & $25(39.7 \%)$ & $34(54.0 \%)$ & $65(46.8 \%)$ & 8.171 & 0.043 \\
\hline Hyperechoic spleen & $16(5.3 \%)$ & $0(0 \%)$ & $1(1.6 \%)$ & $3(4.8 \%)$ & $12(8.6 \%)$ & 8.699 & 0.108 \\
\hline Hepatomegaly & $117(39.0 \%)$ & $14(40.0 \%)$ & $18(28.6 \%)$ & $34(54.0 \%)$ & $51(36.7 \%)$ & 9.140 & 0.027 \\
\hline Renomegaly & $56(18.7 \%)$ & $6(17.1 \%)$ & $8(12.7 \%)$ & $16(25.4 \%)$ & $26(18.7 \%)$ & 11.455 & 0.052 \\
\hline Increased renal echo & $112(37.3 \%)$ & $9(25.7 \%)$ & $10(15.9 \%)$ & $27(42.9 \%)$ & $66(47.5 \%)$ & 21.362 & 0.0001 \\
\hline Lymphadenopathy & $95(31.7 \%)$ & $5(14.3 \%)$ & $16(25.4 \%)$ & $23(36.5 \%)$ & $51(36.7 \%)$ & 8.335 & 0.040 \\
\hline Thickened bowel wall & $12(4.0 \%)$ & $0(0 \%)$ & $0(0 \%)$ & $2(3.2 \%)$ & $10(7.2 \%)$ & 6.692 & 0.005 \\
\hline Fluid-filled bowels & $52(17.3 \%)$ & $6(17.1 \%)$ & $3(4.8 \%)$ & $9(14.3 \%)$ & $34(24.5 \%)$ & 12.285 & 0.006 \\
\hline Thickened GBD wall & $22(7.3 \%)$ & $3(8.6 \%)$ & $2(3.2 \%)$ & $4(6.3 \%)$ & $13(9.4 \%)$ & 2.570 & 0.456 \\
\hline Gallstones & $14(4.7 \%)$ & $3(8.6 \%)$ & $1(1.6 \%)$ & $3(4.8 \%)$ & $7(5.0 \%)$ & 2.696 & 0.422 \\
\hline Enlarged pancreas & $31(10.3 \%)$ & $3(8.6 \%)$ & $3(4.8 \%)$ & $5(7.9 \%)$ & $20(14.4 \%)$ & 5.085 & 0.166 \\
\hline Ascites & $17(5.7 \%)$ & $2(5.7 \%)$ & $1(1.6 \%)$ & $4(6.3 \%)$ & $10(7.2 \%)$ & 2.623 & 0.453 \\
\hline Hypoechoic pancreas & $18(6.0 \%)$ & $1(2.9 \%)$ & $4(6.3 \%)$ & $2(3.2 \%)$ & $11(7.9 \%)$ & 2.984 & 0.817 \\
\hline Hyperechoic liver & $40(13.3 \%)$ & $2(5.7 \%)$ & $8(12.7 \%)$ & $6(9.5 \%)$ & $24(17.3 \%)$ & 7.840 & 0.196 \\
\hline Hypoechoic liver & $7(2.3 \%)$ & $0(0 \%)$ & $0(0 \%)$ & $1(1.6 \%)$ & $6(4.3 \%)$ & 7.840 & 0.196 \\
\hline
\end{tabular}


and $71.2 \%$ v. $28.8 \%$ for females v. males in the Not significant, Mild, Advanced and Severe CD4+ categories respectively).

\section{Sonographic findings}

The Severe CD4+ class had the highest proportion of patients with abnormal abdominal sonographic findings (Table II). However, these sonographic findings were nonspecific to particular pathogens, and none of these patients had a biopsy done so that a definitive diagnosis could be made.

\section{Spleen}

The spleen had the highest frequency of sonographic findings, in which splenomegaly was demonstrated in $133(44.3 \%)$ of the patients (Table III), with significant correlation with their $\mathrm{CD} 4+$ counts. There were $16(5.3 \%)$ patients with hyperechoic splenic parenchyma. These cases of splenic hyperechogenicity could not be attributed to any particular disease entity. Furthermore, splenic hyperechogenicity was found to increase in proportion as the $\mathrm{CD} 4+$ count decreased; however, this was not a statistically significant finding $(p=0.108$; Table III). Other splenic findings included 4 (1.3\%) cases of hyperechoic focal masses (Fig. 1) which were seen only in the severe CD4+ category and were attributed to granulomas.

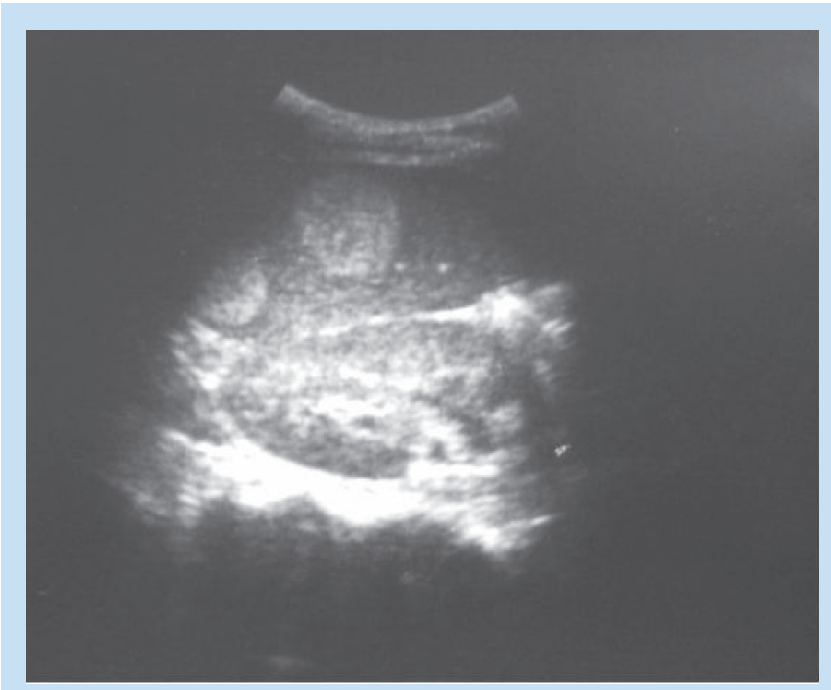

Fig. 1. Ultrasound image showing hyperechoic focal masses in the spleen.

\section{Liver}

Hepatomegaly was the most common liver finding; it was demonstrated in 117 (39\%) patients but did not correlate with their CD4+ counts. Most (253 (84.3\%)) of the patients had normal hepatic echogenicity, whereas $40(13.3 \%)$ patients had hyperechoic parenchyma and $7(2.3 \%)$ patients had a hypoechoic hepatic parenchymal pattern. However, there was no correlation between hepatic echogenicity and CD4+ count (Table III) Furthermore, 3 (1\%) patients had focal hyperechoic hepatic masses which are attributable to hepatic granuloma (Fig. 2), and $1(0.3 \%)$ patient had hepatic calcifications, probably from calcified granulomas (Fig. 3).

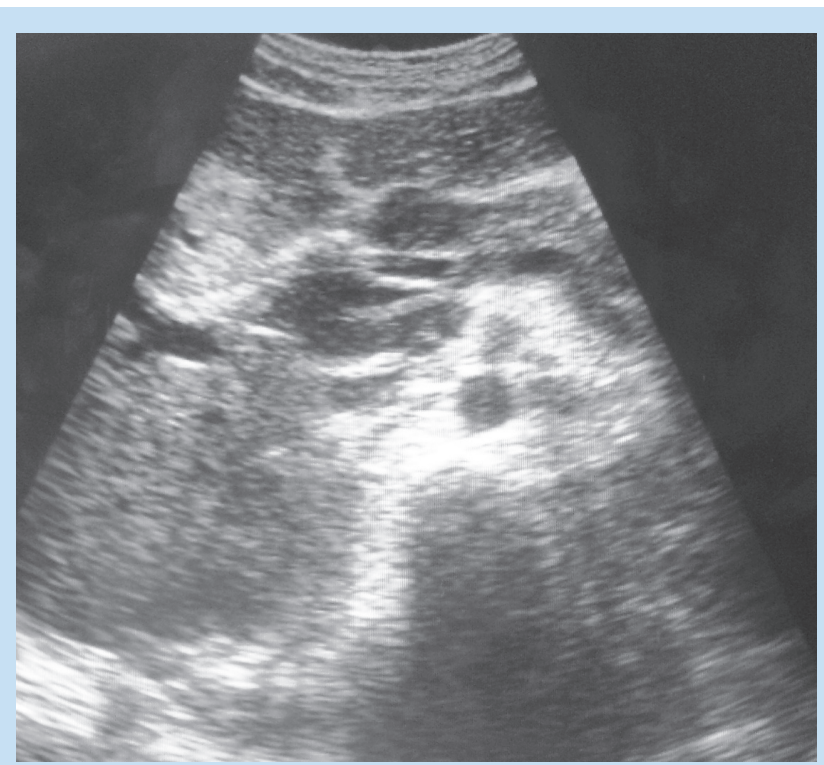

Fig. 2. Ultrasound image of the liver, showing a hyperechoic focal mass in the liver and hepatic hilar lymph node.

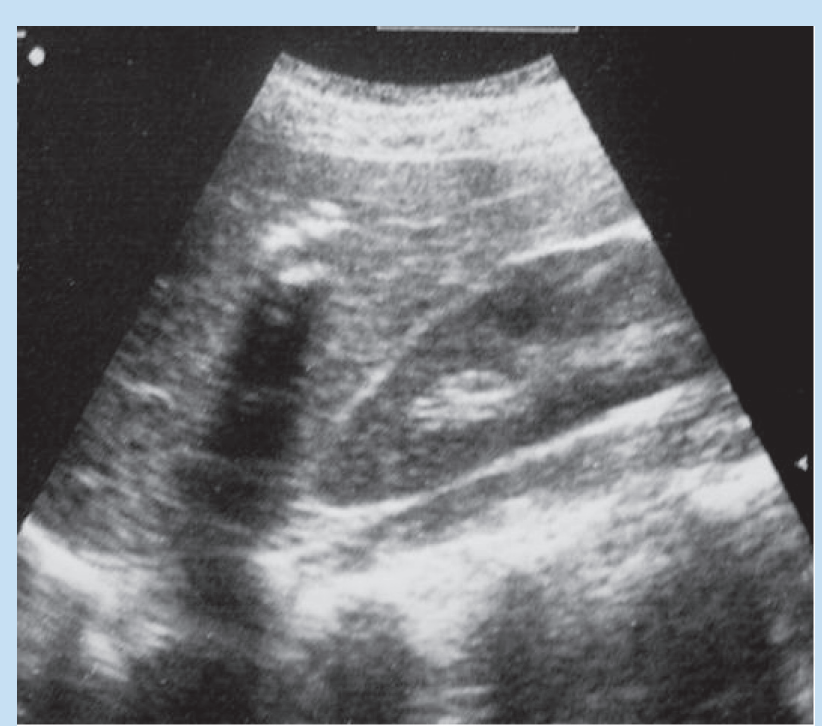

Fig. 3. Ultrasound image of the liver, showing multiple hyperechoic calcifying foci with distal acoustic shadow.

\section{Kidney}

Renomegaly was seen in $56(18.7 \%)$ patients, without significant correlation with their CD4+ counts (Table III). From these cases of renal enlargement, 22 (39.3\%) had bilateral renomegaly, while 23 (41.1\%) had left-sided renomegaly alone, and $11(19.6 \%)$ had right-sided renomegaly only. Hence, left renomegaly occurred more commonly than right-sided renomegaly, which may be because, anatomically, the left kidney generally is larger than the right.

The grading of renal echogenicity was according to that described by Hricak et al. ${ }^{5}$ (grade 0: renal cortical echogenicity < liver parenchymal echogenicity; grade 1: cortex = liver; grade 2: renal sinus >cortex >liver; grade 3: renal sinus $=$ cortex $>$ liver). Normal renal echogenicity was seen 


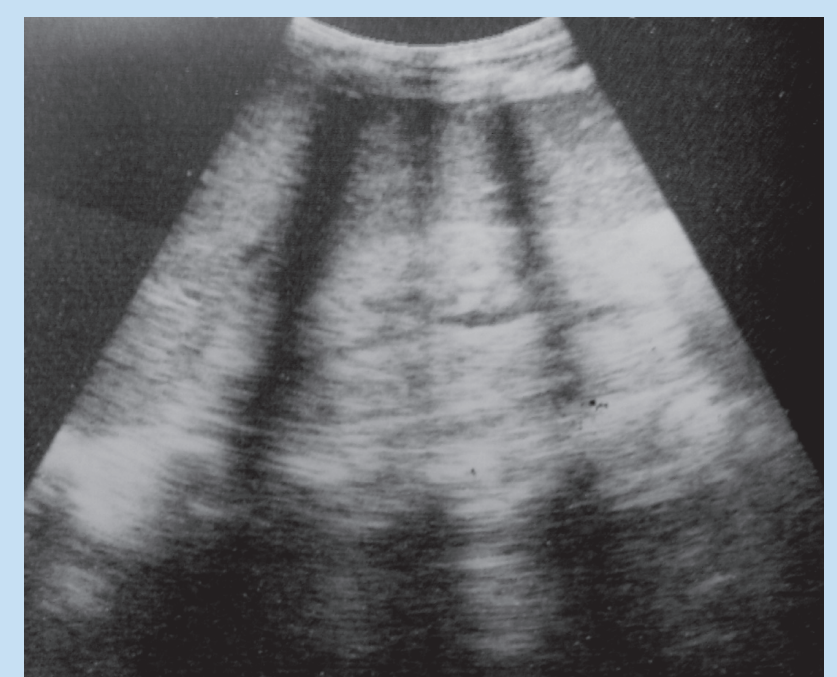

Fig. 4. Ultrasound image of the kidney, showing increased renal cortical echogenicity of Hricak' grade 3.

in $188(62.7 \%)$ patients, while $112(37.3 \%)$ patients had varying grades of renal echogenicity (Table IV, Fig. 4). Patients with depleted CD4+ had a higher proportion of increased cortical echogenicity, whereas the total number of patients with increased renal cortical echogenicity decreased as the grade of cortical echogenicity increased. There were significantly more patients with renomegaly and increased renal echogenicity than those patients with normal-sized kidneys and increased renal echogenicity (53.6\% v. 33.6\%, $p=0.006)$ (Table V).

Other renal findings included $1(0.3 \%)$ case of hydronephrosis, 2 $(0.7 \%)$ cases of renal stones, and $1(0.3 \%)$ case of renal cysts.

\section{Pancreas}

Pancreatic enlargement (Fig. 6) was seen in 31 (10.3\%) patients without correlation with their CD4+ count (Table III). No patient had enlargement involving only the body or the tail of the pancreas. In addition, no focal pancreatic lesion or hypoechogenicity was seen. Most patients in this study (269 (89.7\%)) had normal pancreatic echogenicity; however, all of the $18(6 \%)$ patients with diffuse hypoechoic pancreatic parenchyma had associated pancreatic enlargements. Furthermore, there was no correlation between pancreatic hypoechogenicity and CD4+ count (Table III).

\section{Gallbladder and biliary system}

The gallbladder wall was thickened in 22 (7.3\%) patients, and $14(4.7 \%)$ cases of cholelithiasis were seen; both findings did not correlate with CD4+ count. Biliary duct abnormalities were also not commonly seen. There were $2(0.7 \%)$ cases of biliary duct dilatation (both in the Severe CD4+ class) and 1 case of biliary wall hyperechogenicity. These patients with gallbladder and biliary duct abnormalities were clinically asymptomatic; the clinicians therefore decided in favour of not conducting further evaluations.

\section{Gastrointestinal tract}

There were 52 (17.3\%) patients with fluid-filled bowel loops (Fig. 7), with the Severe class of patients having the highest proportion, but without significant correlation with their CD4+ count (Table III). Bowel walls were thickened in $12(4.0 \%)$ patients. In addition, hyperactive and hypoactive bowels were seen in $11(3.7 \%)$ and $13(4.3 \%)$ of the patients respectively (Table VI), which did not correlate with their CD4+ counts. Varying amounts of ascitic fluids (Fig. 7) were seen in 17 (5.7\%) patients but this did not correlate with their CD4+ counts. These gastrointestinal

Table IV. Distribution of increased renal echogenicity within the CD4+ classes

\begin{tabular}{|c|c|c|c|c|c|}
\hline \multirow{2}{*}{$\begin{array}{l}\text { CD4+ classification } \\
N \text { (\% within class) }\end{array}$} & \multirow[t]{2}{*}{ Normal } & \multicolumn{3}{|c|}{ Renal echogenicity } & \multirow[t]{2}{*}{ Total } \\
\hline & & Grade 1 & Grade 2 & Grade 3 & \\
\hline Not significant & $26(74.3 \%)$ & $5(14.3 \%)$ & $3(8.6 \%)$ & $1(2.9 \%)$ & $35(100 \%)$ \\
\hline Mild & $53(84.1 \%)$ & $7(11.1 \%)$ & $2(3.2 \%)$ & $1(1.6 \%)$ & $63(100 \%)$ \\
\hline Advanced & $36(57.1 \%)$ & $19(30.2 \%)$ & $6(9.5 \%)$ & $2(3.2 \%)$ & $63(100 \%)$ \\
\hline Severe & $73(52.5 \%)$ & $42(30.2 \%)$ & $18(12.9 \%)$ & $6(4.3 \%)$ & $139(100 \%)$ \\
\hline Total & $188(62.7 \%)$ & $73(24.3 \%)$ & $29(9.7 \%)$ & $10(3.3 \%)$ & $300(100 \%)$ \\
\hline
\end{tabular}

\section{Lymph node}

Lymph nodes were seen in 95 (31.7\%) patients in this study, and correlated with their CD4+ count (Table III, Figs 2 and 5). The mean short axis diameter of the lymph nodes was $1.9+0.5 \mathrm{~cm}$, while the minimum short axis diameter was $0.6 \mathrm{~cm}$, and the maximum $2.7 \mathrm{~cm}$. These lymph nodes were seen in the hepatic hilum in $70(73.7 \%)$ patients, para-aortic in 65 (68.4\%), coeliac in 50 (52.6\%), splenic hilum in $15(15.8 \%)$ and in other sites such as the mesenteric and pelvic regions in $5(5.3 \%)$ patients. Many of these lymph nodes were seen at multiple sites.
Table V. Renal size and increased renal echogenicity

\begin{tabular}{|c|c|c|c|}
\hline \multirow{2}{*}{$\begin{array}{l}\text { Renal size } \\
\text { (\% within class) }\end{array}$} & \multicolumn{2}{|c|}{ Renal echogenicity } & \multirow[t]{2}{*}{ Total } \\
\hline & Normal & Increased & \\
\hline Normal & $162(66.4 \%)$ & $82(33.6 \%)$ & $244(100 \%)$ \\
\hline Renomegaly & $26(46.4 \%)$ & $30(53.6 \%)$ & $56(100 \%)$ \\
\hline Total & $188(62.7 \%)$ & $112(37.3 \%)$ & $300(100 \%)$ \\
\hline
\end{tabular}




\section{ORIGINAL ARTICLE}

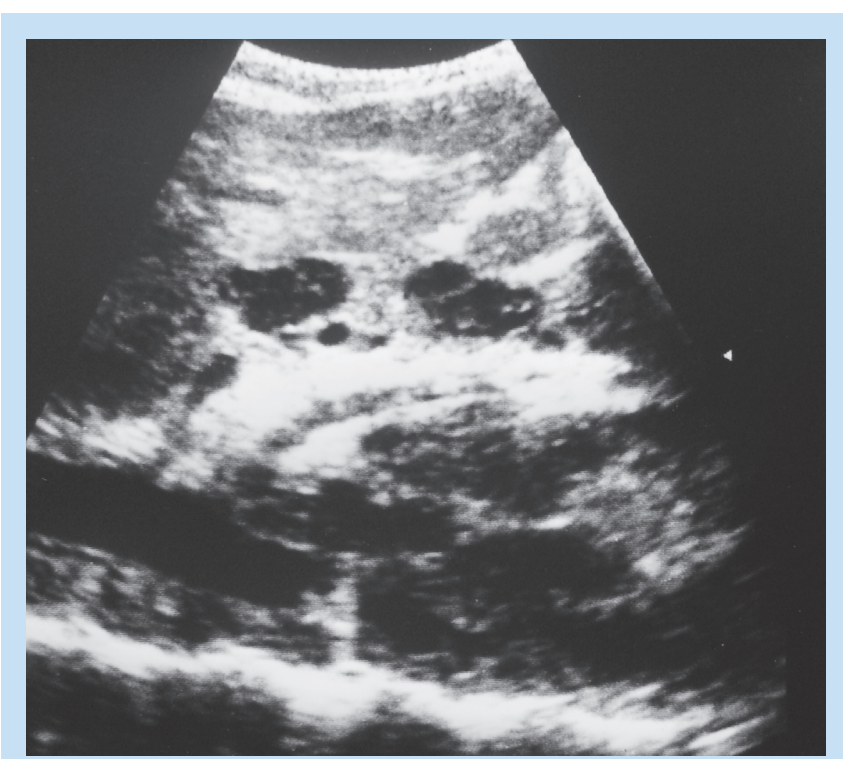

Fig. 5. Ultrasound image of the abdomen, showing multiple lymph nodes - celiac, para-aortic and mesenteric.

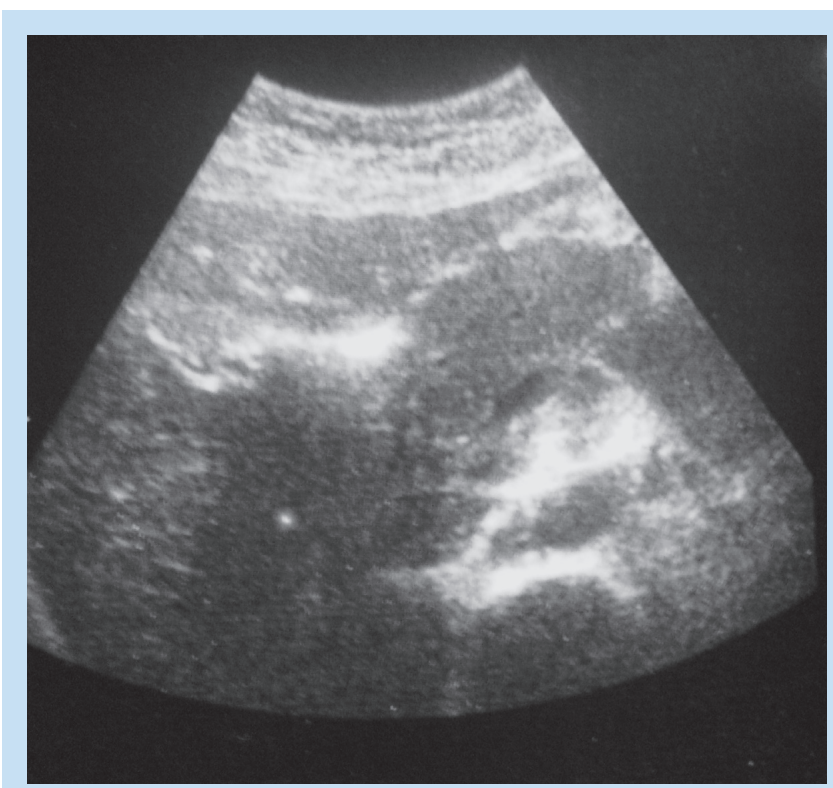

Fig. 6. Ultrasound image showing diffusely enlarged hypoechoic pancreas.

manifestations can be attributed to diarrhoea and enteritis caused by opportunistic pathogens.

\section{Others}

Other findings in this study included 3 cases of left-sided pleural effusions, 2 cases of intra-abdominal abscesses (Fig. 8) and 1 case of anterior abdominal wall abscess (Fig. 9). The latter patient underwent incision and drainage after sonographic diagnosis. Other notable incidental sonographic findings in this study were 2 cases of huge uterine fibroids and a case each of right ovarian complex mass, midline infra-umbilical incisional hernia, and a markedly enlarged prostate gland.

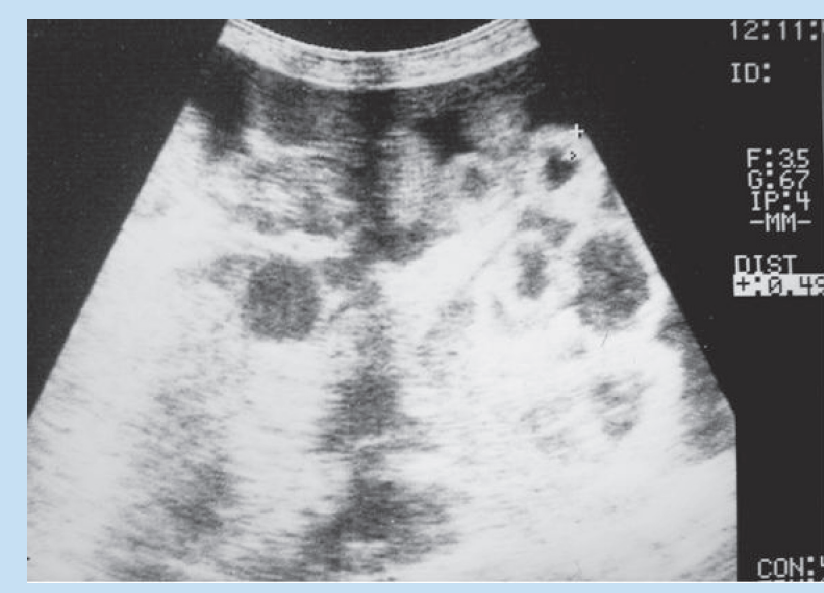

Fig. 7. Abdominal ultrasound image showing thick-walled fluid-filled bowels measuring $4.9 \mathrm{~mm}$ with ascites.

\section{Discussion}

An estimated 23 million people in sub-Saharan Africa are HIV-infected, out of the 33 million living with the disease worldwide. ${ }^{6}$ The HIV virus causes a progressive reduction of cell-mediated immunity, leading to opportunistic infections, neoplasia and even local infection by the virus itself. In many sub-Saharan regions, well-equipped laboratories and radiological equipment are prohibitively expensive and unavailable; consequently, the use of ultrasound in the investigation of these patients has greatly increased because it is affordable, increasingly available, and gives results comparable with computed tomography in competent hands. Furthermore, ultrasound can also be used for monitoring treatment and complications. Unfortunately, the absence of clinicopathological data owing to scarcity of resources in developing countries makes the sensitivity and specificity of ultrasound difficult to assess. ${ }^{8}$

Over $89 \%$ of the patients were within the age range of 18 - 47 years, thereby constituting the majority of the patients in this study population. This age range was described by Adeoye $^{1}$ as the economically productive segment of Nigerian society and also the age group at the greatest risk of HIV/AIDS. However, there were few patients above 57 years old, possibly because most of the older population are less sexually active or promiscuous. However, with the present availability of free/subsidised drugs and therapy, many more elderly HIV-infected patients may be seen in the future, from the HIV-infected youths that survived.

Various abnormal abdominal sonographic findings were seen in $85 \%$ of the 300 patients, which is consistent with the observation made by Smith et al. ${ }^{7}$ that, out of the 95 studies on abdominal sonographic findings in HIV patients which they reviewed, 77 studies documented abnormalities in about $81 \%$ of the patients. The common and uncommon abdominal sonographic abnormalities found in this study are comparable with those documented by other authors. ${ }^{7-9}$ These sonographic abnormalities are non-specific to a particular pathogen or disease entity. ${ }^{7-9}$ However, such findings in the right clinical context and laboratory evaluation will help clinicians to arrive at a diagnosis, leading to better patient care and management.

The frequency of splenomegaly is comparable with that recorded by Yee et al. ${ }^{10}$ in which $45 \%$ of their patients had splenomegaly, com- 
Table VI. Bowel activity v. CD4+ classification

\begin{tabular}{|c|c|c|c|c|}
\hline \multirow{2}{*}{$\begin{array}{l}\text { CD4+ classification } \\
N(\% \text { within class })\end{array}$} & \multicolumn{3}{|c|}{ Gastrointestinal peristalsis } & \multirow[t]{2}{*}{ Total } \\
\hline & Hypoactive & Normoactive & Hyperactive & \\
\hline Not significant & $0(0.0 \%)$ & $34(97.1 \%)$ & $1(2.9 \%)$ & $35(100 \%)$ \\
\hline Mild & $2(3.2 \%)$ & $60(95.2 \%)$ & $1(1.6 \%)$ & $63(100 \%)$ \\
\hline Advanced & $2(3.2 \%)$ & $58(92.1 \%)$ & $3(4.8 \%)$ & $63(100 \%)$ \\
\hline Severe & $9(6.5 \%)$ & $124(89.2 \%)$ & $6(4.3 \%)$ & $139(100 \%)$ \\
\hline Total & $13(4.3 \%)$ & $276(92.0 \%)$ & $11(3.7 \%)$ & $300(100 \%)$ \\
\hline
\end{tabular}

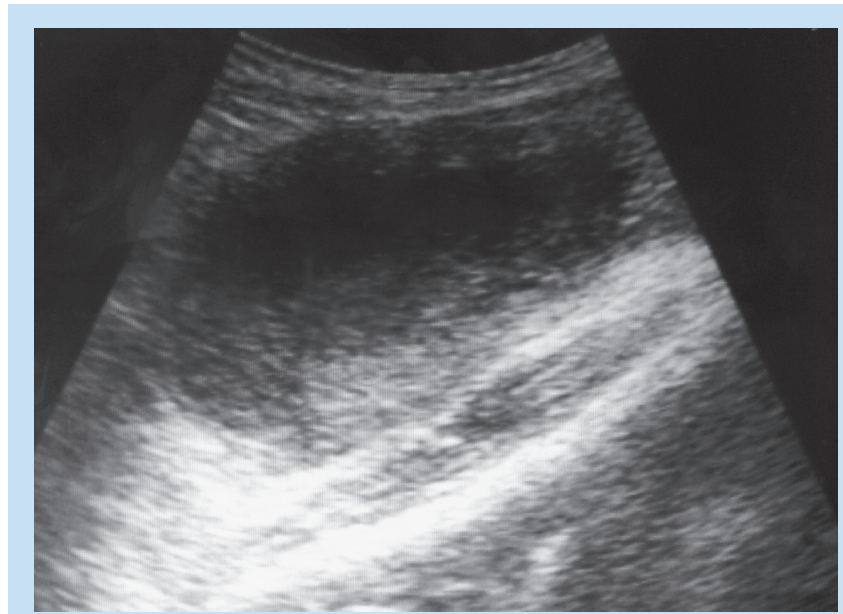

Fig. 8. Abdominal ultrasound image showing an intra-abdominal abscess with echogenic debris within it.

pared with $44 \%$ in this study. Splenomegaly without focal masses is relatively common in the tropics and can have myriads of causes including malaria, septicaemia, typhoid, schistosomiasis, portal hypertension, haemolytic anaemia and tropical splenomegaly.

Hepatomegaly is commonly attributed to either a non-specific response to infection, hepatitis, fatty change, or neoplastic infiltration from lymphoma or Kaposi's sarcoma. ${ }^{11}$ However, hepatosplenomegaly in AIDS patients in the absence of hepatic focal lesions may suggest infection (M. avium intracellulare, malaria or histoplasmosis) rather than lymphoma. ${ }^{12}$

Yee et al. ${ }^{10}$ and Schneiderman $e t a l .{ }^{11}$ attributed the diffuse increased hepatic echogenicity mostly to fatty infiltration or hepatic granulomatosis. However, hepatitis from infection or drugs can cause the observed hyperechoic and also hypoechoic hepatic parenchyma changes. In another study, biopsy-proven cases with similar hyperechoic hepatic lesions to those seen in this study were described as caused by either granuloma or haemangioma.

Renal disease may be caused by the HIV virus itself, secondary infections or the administered drugs. The statistically significant finding of more patients with renomegaly and associated increased cortical echogenicity may be ascribed to HIV nephropathy, which usually causes

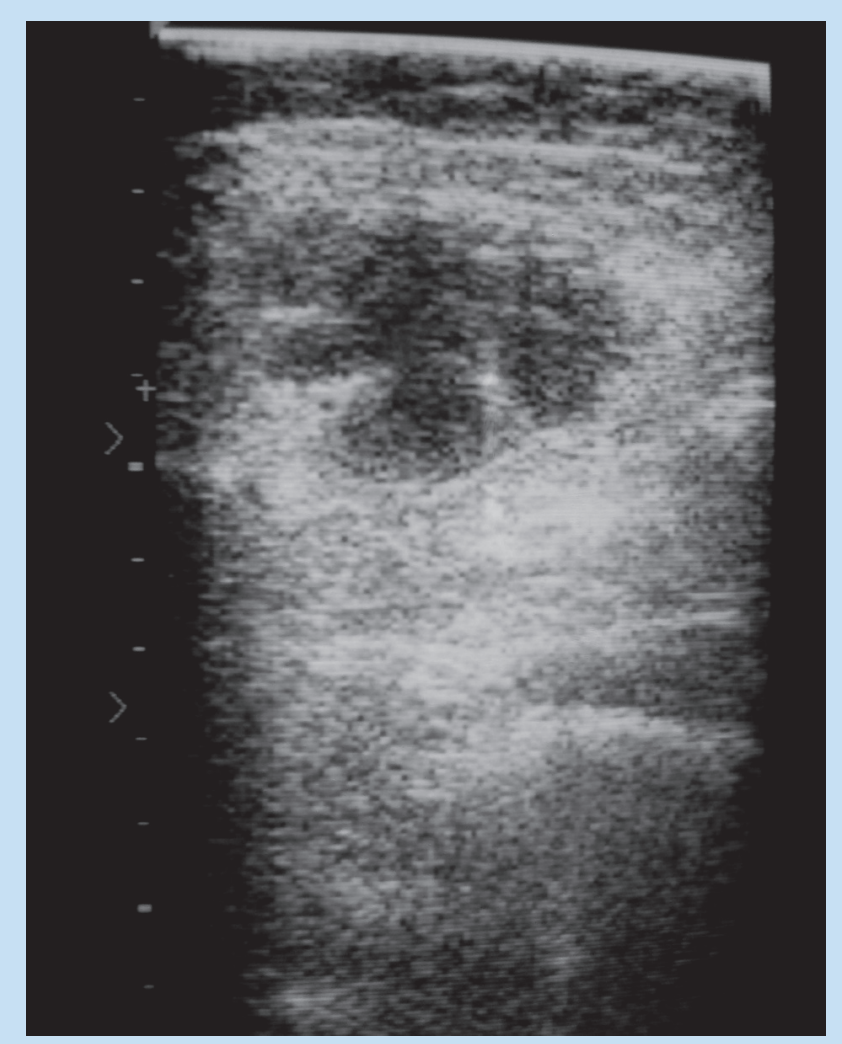

Fig. 9. Abdominal ultrasound image showing an anterior abdominal wall abscess.

diffuse renal cortical echogenicity as well as renal enlargement. ${ }^{13}$ The pattern of increased renal cortical echogenicity seen in this study is similar to that described by some authors as AIDS nephropathy which is shown to occur primarily in black patients. ${ }^{7}$ Surprisingly, Hricak et al..$^{5}$ found no correlation between renal sonographic appearance and the type of renal disease, while Schaffer $e t$ al. ${ }^{13}$ found no definite correlation between the degree of echogenicity and the severity of the renal disease.

However, the abnormal renal sonographic findings in patients with AIDS suggest that even if a patient has no laboratory evidence of renal disease, a baseline renal sonogram could be useful for comparison if the patient ultimately presents with proteinuria or uraemia. ${ }^{13}$ Furthermore, 
there is evidence which suggests that HIV-associated nephropathy will respond dramatically to highly active antiretroviral treatment (HAART) ${ }^{14}$ Hence, prompt detection of AIDS nephropathy and followup sonography may be beneficial to such patients.

Renal stones, which are one of the uncommon findings in this study, can arise in HIV patients on indinavir treatment and can be readily detected on renal ultrasound. However, none of these patients was on indinavir therapy. Hence, the detected nephrolithiasis is a coincidental finding.

Lymph nodes are important components of the immune system that filter and remove foreign particles. In HIV/AIDS patients, lymph nodes are expected to be more active owing to the increased occurrence of infections (and neoplasia). In this study, the frequency of occurrence of visualised lymph nodes correlated with patients' CD4+ counts, which Erdozain et al..$^{15}$ ascribed to the fact that as patients become more immune-compromised, the probability of factors (such as infection and neoplasia) causing lymph node enlargement increases.

None of the demonstrable lymph nodes had focal hypoechoic areas of necrosis, and their short axis diameters were $<3 \mathrm{~cm}$. Consequently, these lymph nodes may be attributed to reactive hyperplasia and would not require further investigations such as biopsy. ${ }^{7}$ Enlarged lymph nodes can involve multiple sites and can be extensive from the diaphragm to the pelvis.

Clinical diagnosis of pancreatitis was made in patients with similar diffuse pancreatic enlargement and hypoechogenicity in another study. ${ }^{7}$ Pancreatitis in HIV patients may be owing to toxoplasmosis, cytomegalovirus, M. avium intracellulare or antiretroviral treatment. The reason why there was no localised pancreatic enlargement involving the body or tail but involving only the head of the pancreas in 13 patients is unknown.

Sonographic findings in the gallbladder and biliary tract were not common, and were for unknown reasons lower than those recorded by some authors. ${ }^{7,9}$

The gastrointestinal findings in this study (thickened bowel walls, fluid-filled bowels, ascites, hyperactive and hypoactive bowels - which are features of gastrointestinal infections) were mainly demonstrated in the small intestine, and their high incidence in the Severe CD4+ class of patients may be attributed to the increased frequency of opportunistic gastrointestinal infections with depressed immunity since immunosuppression impairs gastric acid secretion and peristalsis, which are non-specific host defence mechanisms. Hence, HIV-infected patients have gastric secretory failure, altered intestinal motility, impaired local immunoglobulin A and cellular immune responses, ${ }^{16}$ which predisposes the gastrointestinal tract to increased bacterial colonisation, leading to malabsorption, diarrhoea and opportunistic infections.

\section{Conclusion}

The findings in this study demonstrate that a myriad of sonographic abnormalities can appear in the abdomens of HIV/AIDS patients. Splenomegaly, hepatomegaly, increased renal cortical echogenicity, lymphadenopathy, thickened bowel walls and fluid-filled bowels correlated with CD4+ counts. Most of the sonographic abnormalities did not correlate with CD4+ counts, indicating that there is no direct association between the infection stage, the $\mathrm{CD} 4+$ count and ultrasound findings.

To conclude: ultrasound scans should be used as a routine baseline investigation as well as in the follow-up of HIV-infected patients.

We thank GOG Awosanya, AO Akhigbe, OT Ogbeide, KC Eze, JE Ikubor, Eu Eze and PFI Irabor for their technical support, manuscript proofreading and general assistance and advice.

1. Adeoye S. Sexual behaviour, perception of HIV/AIDS and condom use among commercial motorcyclists in Benin City. Nig Postgrad Med J 2005; 12(4): 262-265.

2. World Health Organization. Summary country profile for HIV/AIDS treatment scale up - Nigeria. Geneva: World Health Organization, Dec 2005

3. Moore EH, Gaensler EHL, Imalingat B, Reeves P, Wachira MW. The acquired immunodeficiency syndrome (AIDS) in the tropics. In: Palmer PES, Reeder MM, eds. The Imaging of Tropical Diseases. Berlin: Springer, 2001: 681-740.

4. World Health Organization. WHO case definitions of HIV for surveillance and revised clinical staging and immunological classification of HIV-related disease in adults and children. Geneva: World Health Organization, 2006: 16

5. Hricak H, Cruz C, Romanski R, et al. Renal parenchymal disease; sonologic-histologic correlation. Radiology 1982; 144: 141-147.

6. Satcher D. The global HIV/AIDS pandemic. JAMA 1999; 281: 1425-1479.

7. Smith FJ, Mathieson JR, Cooperberg PL. Abdominal abnormalities in AIDS: detection at US in a large population. Radiology 1994; 192: 691-695.

8. Tshibwabwa ET, Mwaba P, Bogle-Taylor J, Zumla A. Four-year study of abdominal ultrasound in 900 Central African adults with AIDS referred for diagnostic imaging. Abdom Imaging 2000; 25(3): 290-296.

9. Obajimi MO, Atalabi MO, Igbole GI, et al. Abdominal ultrasonography in HIV/AIDS patients in SouthWestern Nigeria. BMC Med Imaging 2008; 8: 5-10.

10. Yee JM, Raghavendra BN, Horii SC, Ambrosino M. Abdominal sonography in AIDS: a review. J Ultrasound Med 1989; 8: 705-714.

11. Schneiderman DJ. Hepatobiliary abnormalities of AIDS. Gastroenterol Clin North Am 1988; 17: 615-630

12. Townsend RR. CT of AIDS-related lymphoma. Am J Roentgenol 1991; 156: 969-974.

13. Schaffer RM, Schwartz GE, Becker JA, Rao TKS, Shih YH. Renal ultrasound in acquired immune deficiency syndrome. Radiology 1984; 153: 511-513.

14. Brook MG, Miller RF. HIV associated nephropathy: a treatable condition. Sex Transm Infect 2001; 77: 97-100.

15. Erdozain E, Gonzalez M, Pintado V, Munoza JF, Castillo P. Abdominal echography in patients with immunodeficiency virus (HIV) infection. Diagnostic usefulness in the evaluation of associated medicosurgical procedure. Ann Med Intern 1995; 12: 115-121.

16. Haller JO, Cohen HL. Gastrointestinal manifestation of AIDS in children. Am J Roentgenol 1994; 162: 387-393. 